\title{
The role of diallyl thiosulfinate associated with nuciferine and diosgenin in the treatment of premature ejaculation: A pilot study
}

\author{
Tommaso Cai ${ }^{1}$, Andrea Cocci ${ }^{2}$, Gianmartin Cito ${ }^{2}$, Bruno Giammusso ${ }^{3}$, Alessandro Zucchi ${ }^{4}$, \\ Francesco Chiancone ${ }^{5}$, Maurizio Carrino ${ }^{5}$, Francesco Mastroeni ${ }^{6}$, Francesco Comerci ${ }^{7}$, \\ Giorgio Franco $^{8}$, Alessandro Palmieri ${ }^{9}$ \\ ${ }^{1}$ Department of Urology, Santa Chiara Regional Hospital, Trento, Italy; \\ ${ }^{2}$ Department of Urology, University of Florence, Florence, Italy; \\ ${ }^{3}$ Department of Andrology, Policlinico Morgagni, Catania, Italy; \\ ${ }^{4}$ Department of Urology, University of Perugia, Perugia, Italy; \\ ${ }^{5}$ Department of Urology, Cardarelli Hospital, Naples, Italy; \\ ${ }^{6}$ Department of Urology, Azienda Ospedaliera Papardo, Messina, Italy \\ ${ }^{7}$ Urologist, Bologna, Italy; \\ ${ }^{8}$ Department of Urology, Sapienza University of Rome, Rome, Italy; \\ ${ }^{9}$ Department of Urology, University of Naples, Federico II, Naples, Italy.
}

\begin{abstract}
Summary Objective: To assess the efficacy and safety of an association of diallyl thiosulfinate with nuciferine and diosgenin in the treatment of a group of patients suffering from premature ejaculation (PE), primary or secondary to erectile dysfunction (ED).

Materials and methods: From July 2015 to October 2016, 143 patients (mean age 25.3; range 18-39) affected by PE completed the study and were finally analyzed in this phase I study. All patients, after clinical assessment and laboratory evaluation were asked to take an association of diallyl thiosulfinate with nuciferine and diosgenin as oral tablet, once a day, on alternate days, for three months. At the baseline and after three months of treatment, each patient was asked to complete the following questionnaires: International Index of Erectile Function (IIEF-5), Premature Ejaculation Diagnostic Tool (PEDT), Male Sexual Health Questionnaire (MSHQ).

Results: A statistical significant improvement in terms of erectile function, comparing the IIEF-5 value at baseline and follow-up visit was found (respectively IIEF-5: 8.7 vs 14.01; $p<0.001)$. Moreover, at follow-up visit, $97 / 143$ men $(67.8 \%)$ referred a subjective improvement of the erection quality and a better control of the ejaculation (PROs). The IELT improved too between the baseline evaluation and the follow-up visit $(p<0.001)$.

Conclusion: In conclusion, our study, even if supported by preliminary results, showed how Diallyl Thiosulfinate, Nuciferine and Diosgenin is able to improve the control of ejaculation in patients suffering from $P E$, primary or secondary to $E D$ without any significant adverse effects.
\end{abstract}

KeY WORDS: Diosgenin; Muciferine; Thiosulfinate; Plant extracts; Premature ejaculation; Treatment.

Submitted 6 December 2017; Accepted 12 December 2017

\section{INTRODUCTION}

Premature ejaculation (PE) is defined as uncontrolled ejaculation either before or shortly after sexual penetration (1). It is a very common male sexual dysfunction with prevalence rates of $20-30 \%$, probably affecting every man at some point in his life $(2,3)$. It may result in unsatisfactory sexual intercourse for both partners, decreasing sexual self-confidence and self-esteem and resulting in an overall reduction of quality of life (QoL) (4). Lifelong PE occurs within 30-60 seconds after vaginal penetration with nearly every coitus, interesting about $>85 \%$ of men affected by this dysfunction, whereas about $10-20 \%$ of men ejaculate within 1-2 minutes (5). Acquired PE is commonly due to sexual performance anxiety (6), psychological or relationship problems (6), erectile dysfunction (ED) (7), occasionally prostatitis (8), hyperthyroidism (9), or during withdrawal/detoxification from prescribed (10) or recreational drugs (11). In addition, men with this dysfunction are usually older, have a higher mean body max index and a greater incidence of comorbid disease and ED associated to lifelong, variable and subjective PE $(12,13)$. Although the pathophysiologic mechanism of lifelong PE is not fully understood, it is shown that psychosocial, as well as organic factors play a key role in the aetiology (14-16). Several studies demonstrated that patients with PE have more dorsal penile nerves, increasing glands penile hypersensivity and hyperexcitability (17). Moreover, they also usually have other abnormal autonomic reflex pathways for the ejaculatory process, including shorter bulbo-cavernosal latency time and higher bulbo-cavernosal evoked potentials $(18,19)$. For this reason, pharmacologic therapy that reduce glands penile hypersensivity should be effective for the treatment of PE. Medications, counselling and sexual techniques, that may delay ejaculation, can help the patients to improve sexual intercourse. However, a combination of these factors could be the best choice of treatment to improve efficacy and minimize relapse (20). Behavioural therapy essentially include the "stop-start program" developed by Semans (21), and its modification, the "squeeze" technique, pro-

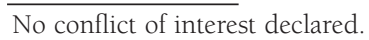


posed by Masters and Johnson (22). All these approaches need the partner's cooperation for a long time, resulting often difficult in the long term (23). Pharmacological therapy includes: selective serotonin reuptake inhibitors (SSRIs) antidepressants (24); dapoxetine (25); anaesthetic creams (26) and phosphodiesterase type 5 inhibitors (PDE5i) (27). Also some natural compounds (i.e. Satureja montana, Tribulus terrestris, Phyllantus emblica) proved to be efficient in improving sexual quality of life in patients with PE (28). The role of nutraceuticals seems, then, interesting in the management of PE.

The aim of this study was to assess the efficacy and safety of Diallyl Thiosulfinate, Nuciferine and Diosgenin (CAMPEDEX $-5^{\circledR}$ ) in the treatment of patients affecting by PE, primary or secondary to ED.

\section{MATERIALS AND METHOdS}

\section{Study design}

From July 2015 to October 2016, we enrolled, in a pilot study, a total of 154 patients, affecting by PE, primary or secondary to ED. Each patient signed a written fully informed consent statement before being enrolled in the study and accepted the trial voluntarily. After enrolment, all patients were asked to take CAMPEDEX-5 ${ }^{\circledR}$ oral tablet, once a day, on alternate days, for three months (Figure 1). After 30 days all contacted by phone in order to be sure about the adherence to study protocol. After 3 months, all patients were evaluated with clinical evaluation and questionnaires in order to test the treatment efficacy and safety.

\section{Inclusion and exclusion criteria}

All men had been in stable, monogamous, heterosexual relationship with at least one sexual intercourse a week with a compliant female partner. Exclusion criteria were: age $<18$ years, history of a psychiatric or neurological disorder, alcohol or drug abuse, previous genitourinary system trauma or surgery, spinal cord injury, previous radiotherapy, thyroid diseases, hypogonadism, currently taking a drug known to affect sexual function, such as hormonal therapy for prostate cancer.

\section{Baseline evaluation}

The aetiology of PE was determined, in all cases, by a complete in-depth medical history, physical examina- tion, hormonal evaluation and patient self-administered questionnaires on sexual function, even submitted to the partners. All the patients received a urological visit that considered the development of external genitalia, the presence of phimosis and/or prepuce anomalies. At the baseline, we collected the following hormonal profile from all patients: serum follicle-stimulating hormone (FSH) concentrations, prolactin levels, thyroid stimulating hormone (TSH), luteinizing hormone (LH) and blood total testosterone (TT). Each patient was asked to complete the following questionnaires: International Index of Erectile Function (IIEF-5), Premature Ejaculation Diagnostic Tool (PEDT) and Male Sexual Health Questionnaire (MSHQ).

\section{Questionnaires and PE status evaluation}

The IIEF-5 scale was used in order to evaluate the erectile function in the last six months considering the severity of ED, classified as follow: severe (IIEF- $5 \leq 7$ ), moderate (IIEF- 5 between 8 and 11), mild-moderate (IIEF-5 between 12 and 16), mild (IIEF-5 17-21), absent (IIEF5 between 22 and 25). PEDT help to identify men who may have a problem with ejaculating too soon during sexual activity, considering the ejaculation as the release of semen after penetration. PEDT investigated the severity of PE, classified as follow: absence of PE (PEDT $\leq 8)$, probably PE (PEDT 9-10), certainly present PE (PEDT $\geq 11$ ) (29). MSHQ-EjD is an abridged version of the 25 item MSHQ, seen as a validated, self-administered instrument for assessing the primary domains of erection, ejaculation, and sexual satisfaction in aging men. MSHQ-EjD described three ejaculatory function items, investigating force ("in the past month, how would you rate the strength or force of your ejaculation?"), volume item ("in the past month, how would you rate the amount or volume of semen or fluid when you ejaculate?"), frequency item ("in the past month, how often have you been able to ejaculate or "cum" when having sexual activity?), and one ejaculation bother item ("if you had any ejaculation difficulties or have been unable to ejaculate, have you been bothered by this?") (30). Ejaculatory function score, which is the sum of the ordinal responses to the three items, ranges from 1 to 15 , while bother scores ranges from 0 to 5 .

Furthermore, we collected the Intravaginal Ejaculation Latency Time (IELT), as the time from vaginal penetration until ejaculation (31). It was timed on a stopwatch by 'start' (penetration) to 'stop' (ejaculation). IELT was asked

\section{Figure 1.}

The figure shows the study schedule.

IIEF-5: International Index of Erectile Function; PEDT: Premature Ejaculation Diagnostic Tool; MSHQ-EjD: Male Sexual Health Questionnaire; IELT: Intravaginal ejaculation latency time; PROs: Patients-Reported Outcomes.

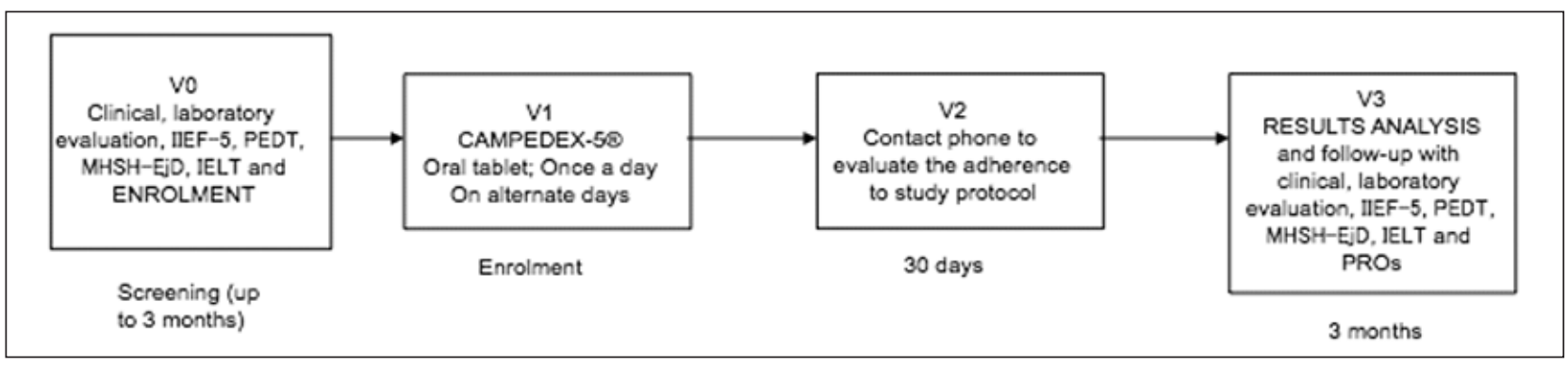


both to men and their partners, trying to be honest in recording the time and it was calculated as mean from that perceived by the men and that perceived by partners. They were instructed to calculate and record the exact time after ejaculation.

\section{Follow-up evaluation}

At 3 months follow-up evaluation, all patients underwent urological examination and the same laboratory and questionnaires evaluation as at the baseline time.

In addition, we evaluated the Patients-Reported Outcomes (PROs). PROs are defined as a report that comes directly from the patient about the status of his health condition without amendment or interpretation of the patient's response by a clinician or anyone else (32).

The "outcomes" in PRO were interpreted broadly to reflect a variety of information reported directly by the patient, investigating the presence of a subjective improvement of erection quality and a positive change of the control of PE. Also health-related QoL, functional status and treatment adherence were collected.

\section{Outcome measures and statistical analysis}

All clinical, laboratory and parameters and questionnaires were compared at the baseline (before to begin treatment) and at follow-up visit (after three months of treatment). All statistical analysis was performed using the IBM SPSS version 20.0 (SPSS Inc, Chicago, IL, USA). $\mathrm{P}<0.05$ was considered statistically significant.

\section{RESULTS}

Of all 154 men enrolled, 143 patients completed the study voluntarily, 11 patients withdrew the study for personal reasons. 81/143 men (56.6\%) had a clinical presentation of lifelong PE, while 62/143 men (43.3\%) presented a secondary PE, caused by sexual performance anxiety, psychological or relationship problems and occasionally prostatitis. 44/143 patients (30.7\%) had ED associated. The mean age was 25.3 years old (range 18-39). At baseline and follow-up visit, all hormonal parameters collected were in the normal range (Table 1). How shown in Table 2 , at the follow-up visit, the patients shown a statistical significant improvement in terms of erectile function, comparing the IIEF- 5 value at baseline and follow-up visit

\section{Table 1.}

Clinical and laboratory characteristics of all analyzed patients.

\begin{tabular}{|lc|}
\hline \multicolumn{2}{|l|}{ Demography, clinical and laboratory characteristics at baseline } \\
\hline Enrolled patients $\mathrm{n}^{\circ}$ & 143 \\
\hline Age (mean) (range) & $25.3(18-39)$ \\
\hline Clinical presentation & 81 \\
Primary PE & 62 \\
Secondary PE & 44 \\
Associated ED & 14.3 \\
\hline Total testosterone (ng/ml) & 5.3 \\
\hline FSH (IU/L) & 6.8 \\
\hline LH (IU/L) & 2.3 \\
\hline TSH (mlU/L) & 7.8 \\
\hline Prolactin (ng/ml)
\end{tabular}

Table 2.

Outcome variables before and after treatment.

\begin{tabular}{|lccc|}
\hline Outcomes variable & Baseline & Follow-up & $\mathbf{P}$ \\
\hline IIEF-5 (mean score) & 8.7 & 14.01 & $<0.001$ \\
\hline PEDT (mean score) & 15 & 10 & $<0.1$ \\
\hline $\begin{array}{l}\text { MHSQ-EjD } \\
\text { (ejaculatory function mean score) }\end{array}$ & 9 & 12 & $<0.1$ \\
\hline MHSQ-EjD & 5 & 3 & $<0.1$ \\
\hline (bother mean score) & 45.5 & 123.7 & $<0.001$ \\
\hline IELT (mean score) [min] & & & \\
\hline
\end{tabular}

(respectively IIEF-5: 8.7 vs 14.0; $<<0.001$ ). Median total PEDT score changed from 15 (baseline) to 10 (follow-up). Regarding MSHQ-EjD, the majority of patients reported, at the end of treatment, a reduction of awkwardness during sexual intercourses (bother score: 5 vs 3 ) and an improvement of ejaculatory function disorders (ejaculatory function score: 9 vs 12). IELT shown a clean improvement, increasing significantly from $45.5 \mathrm{sec}$ to $123.7 \mathrm{sec}$, respectively at baseline and follow-up visit $(\mathrm{p}<0.001)$ (Figure 2). Moreover, at follow-up visit, of all 143 patients, 97/143 men (67.8\%) referred a subjective improvement of the erection quality and a better control of the ejaculation (PROs). During the study, no treatment emergent adverse events occurred, let alone some patients interrupted the drug assumption for concomitant therapies or co-morbidities.

\section{Discussion}

\section{Main finding}

In this study, we demonstrated how the assumption of organic compounds derived from natural substances is able to improve the quality of erections and the control of ejaculation, increasing the mean ejaculation latency time. In particular, we tested the following combination of Diallyl Thiosulfinate (20 mg), Nuciferine (137.5 mg) and Diosgenin (45 mg).

\section{Results in the context of previous studies}

Thiosulfinates are allylsulfide compounds including allicin, diallyldisulfide, diallyltrisulfide, S-allylmercaptocysteine and S-allylcysteine, a class of phytochemical organosulfurs found essentially in garlic and other vegetables from Alliumspecies (33). Allicin, or Diallyl Thiosulfinate, is the most important and representative active ingredient of garlic (Allium sativus). This substance is formed when the clumps that make up the bulbs are cut, chewed or otherwise crushed. Following these mechanical actions, an enzyme, called allinase, releases from the vacuolary juices, acting on an aminoacid, the allyl, transforming it into allicin. Diallyl Thiosulphinate can increase the concentration of intracellular glutathione, a substance consisting of three amino-acids (cysteine, glutamic acid and glycine). Through a series of reactions, cysteine is transformed in hydrogen sulphide (H2S) produced at the arterial level with particular reference to peripheral circulation. H2S acts as a real proerogenous gaseous mediator which, through the opening 
Figure 2.

The figure shows the results at the follow-up evaluation by using questionnaires before (pre) and after (post) treatment. IIEF-5: International Index of Erectile Function; PEDT: Premature Ejaculation Diagnostic Tool; MSHQ-EjD: Male Sexual Health Questionnaire; IELT: Intravaginal ejaculation latency time; PROs: Patients-Reported Outcomes.

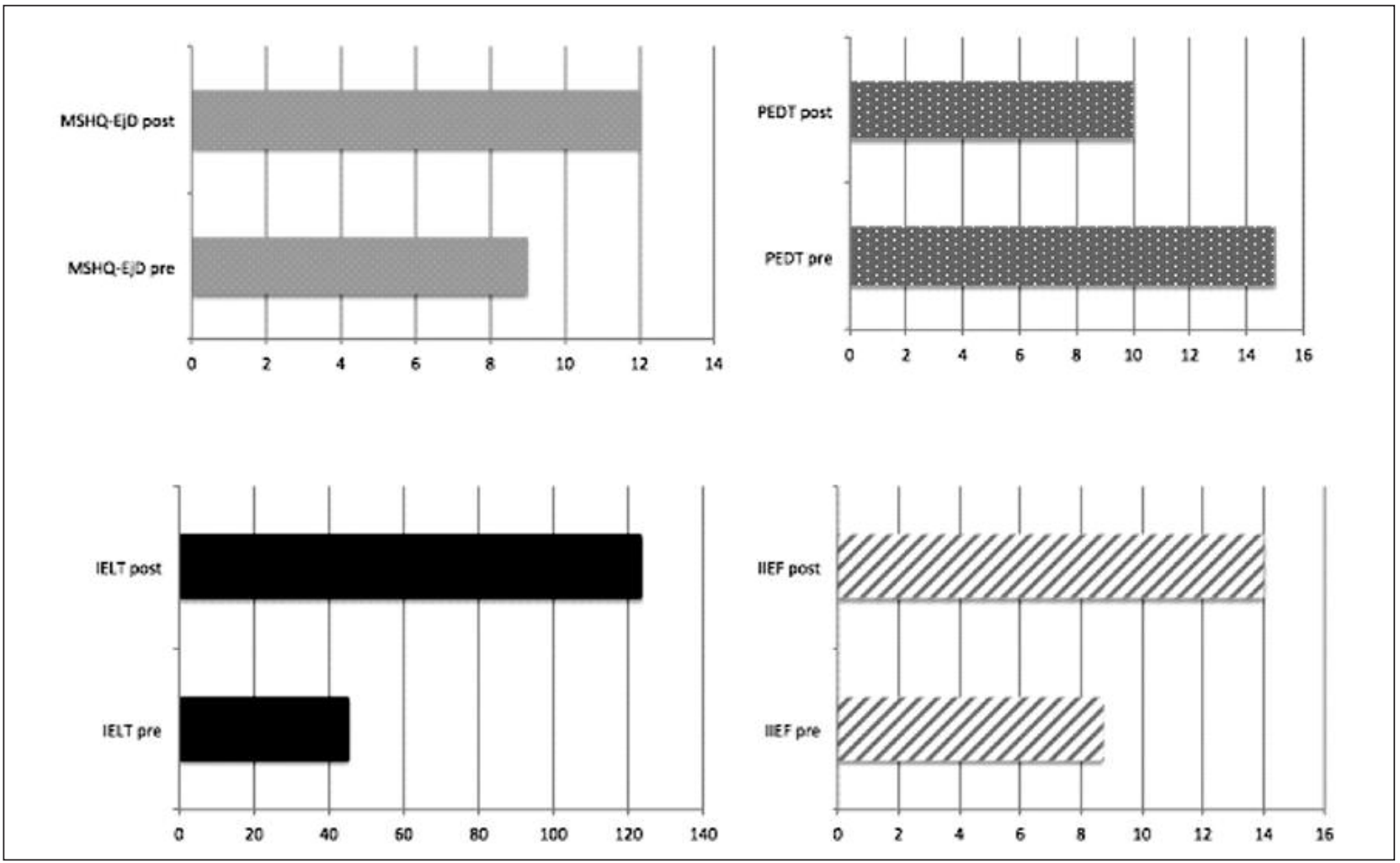

of potassium channel ATP, resulting in increased $\mathrm{CA}^{+}$ ion transfer, produces through a precise hyperpolarization mechanism on smooth muscle cells, a relaxation effect on them. The relaxation of the peripheral smooth muscle also causes the recall of blood resulting in an erection phenomenon (34).

Diosgenine is the most important active principle of Dioscorea (Dioscorea villosa), also known as wild yam, a plant belonging to the Dioscoreacee family. It is therefore an important precursor of steroids, present in nature in the form of diocin glycoside or other heterosides that are then hydrolysed in acidic environments and has therefore always been used by the pharmaceutical industry as a raw material for the production of hormones, such as DHEA. Small amounts of DHEA are also produced by testicles, ovaries and glial cells. DHEA circulates bloodstream especially as a sulfate (DHEA-S); in the plasma about $80 \%$ of this sulphate is bound to albumin, while the remaining $20 \%$ is linked to lipoproteins. The amount of DHEA contained in the human body is related to the age of the subject. After birth there is a significant decrease in plasma levels of DHEA. Starting at five years, levels are rising again to reach the peak of the age of twenty-five; From the age of twenty-five, there is a progressive decrease in the concentration of DHEA in the body; this decline begins to become rapid from forty years. At eighth decade, the level does not exceed 10\% of the maximum reached twenty-five years. Because of this decrease in plasma levels with age, many authors have suspected that DHEA was heavily involved in aging-regulating processes, so DHEA administration was recommended as an anti-aging method. DHEA appears to be involved in many biological functions including sexual activity regulation and stimulation, myelin production and activation of the G6PD-H enzyme that helps to reduce fat cells (35). Nuciferine is an alkaloid extracted from the plants Nymphaea caerulea and Nelumbo nucifera. It has a pharmacological profile of action associated with dopamine receptor blockade. Specifically, Nuciferine is a partial antagonist of D2-like receptor, a subtype of Dopamine receptors which activation is associated with ejaculation and micturition stimulus (36).

It induces sedation, hypothermia, ptosis, and catalepsy, if present in higher doses; it inhibits spontaneous motor activity, conditioned avoidance response, amphetamine toxicity and stereotypy. A clinical trial on rats showed that Nuciferin, at doses ranging from 25 to $50 \mathrm{mg}$ per $\mathrm{kg}$ intraperitoneal, is able to produce sedation levels from moderate to marked and ptosis.

The authors have therefore concluded that probably Nuciferin acts by blocking dopaminergic receptors: in a further study it has been shown that this substance is able to inhibit amphetamine-induced stereotype, which, as is known, is mediated by the stimulation of dopaminergic receptors (36).

This present study showed that IELT significantly increased after treatment with CAMPEDEX-5 ${ }^{\circledR}$.

Moreover, Diallyl Thiosulfinate, Nuciferine and Diosgenin 
proved effective in improving the quality of erections, increasing significantly IIEF-5. Topical agents, for the treatment of PE, can reduce the hypersensivity of the glands penis, but can induce localized irritation, including pain, burning, delayed ejaculation and loss of penile sensation (37). Furthermore, in our study, the treatment with CAMPEDEX-5 ${ }^{\circledR}$ was well tolerated by all patients, no showing any side effect reported during the period study. However, the limitation of the study is related to the methodology used, as it was not a case-control study. More studies should be carried out to clarify the precise role of the active ingredients in CAMPEDEX-5 ${ }^{\circledR}$ and their interactions.

\section{Conclusions}

Our study, though based on results obtained from a pilot study, shown how Diallyl Thiosulfinate, Nuciferine and Diosgenin are able to improve the control of ejaculation in patients suffering from PE, primary or secondary to ED, significantly improving IIEF and IELT rates. The drug had thus proven to be safe and effective in the treatment of PE, without having clinically relevant side effects.

\section{REFERENCES}

1. Becker JV, Stinson JD. Premature ejaculation section of Human sexuality and sexual dysfunctions. In RE Hales et al., eds., The American Psychiatric Publishing Textbook of Psychiatry, $5^{\text {th }}$ ed., 2008; pp. 711-728. Washington, DC: American Psychiatric Publishing.

2. Laumann EO, Nicolosi A, Glasser DB, et al. Sexual problems among women and men aged 40-80 y: prevalence and correlates identified in the Global Study of Sexual Attitudes and Behaviors. Int J Impot Res. 2005; 17:39-57.

3. Porst H, Montorsi F, Rosen RC, et al. The Premature Ejaculation Prevalence and Attitudes (PEPA) survey: prevalence, comorbidities, and professional help-seeking. Eur Urol. 2007; 51:816-823

4. Rosen RC, Althof S. Impact of premature ejaculation: the psychological, quality of life, and sexual relationship consequences. J Sex Med. 2008; 5:1296-1307.

5. Waldinger MD. History of Premature Ejaculation. In: Jannini E, McMahon CG, Waldinger MD, editors. Premature Ejaculation. From Etiology to Diagnosis and Treatment. Springer-Verlag Mailand, 2013:5-24.

6. Hartmann U, Schedlowski M, Krüger TH. Cognitive and partnerrelated factors in rapid ejaculation: differences between dysfunctional and functional men. World J Urol. 2005; 23:93-101.

7. Laumann EO, Nicolosi A, Glasser DB, et al. Sexual problems among women and men aged 40-80 y: prevalence and correlates identified in the Global Study of Sexual Attitudes and Behaviors. Int J Impot Res. 2005; 17:39-57

8. Screponi E, Carosa E, Di Stasi SM, et al. Prevalence of chronic prostatitis in men with premature ejaculation. Urology. 2001; 58:198-202.

9. Carani C, Isidori AM, Granata A, et al. Multicenter study on the prevalence of sexual symptoms in male hypo- and hyperthyroid patients. J Clin Endocrinol Metab. 2005; 90:6472-9.

10. Adson DE, Kotlyar M. Premature ejaculation associated with citalopram withdrawal. Ann Pharmacother. 2003; 37:1804-6.
11. Peugh J, Belenko S. Alcohol, drugs and sexual function: a review. J Psychoactive Drugs. 2001; 33:223-32.

12. Basile Fasolo C, Mirone V, Gentile V, et al. Premature ejaculation: prevalence and associated conditions in a sample of 12,558 men attending the andrology prevention week 2001--a study of the Italian Society of Andrology (SIA). J Sex Med. 2005; 2:376-82.

13. Porst H, McMahon CG, Althof SE, et al. Baseline characteristics and treatment outcomes for men with acquired or lifelong premature ejaculation with mild or no erectile dysfunction: integrated analyses of two phase 3 dapoxetine trials. J Sex Med. 2010; 7:223142.

14. Xin ZC, Choi YD, Seong DH, Choi HK. Sensory evoked potential and effect of SS-cream in premature ejaculation. Yonsei Med J. 1995; 36:397-401.

15. Xin ZC, Chung WS, Choi YD, et al. Penile sensitivity in patients with primary premature ejaculation. J Urol. 1996; 156:979-981.

16. Paick JS, Jeong H, Park MS. Penile sensitivity in men with premature ejaculation. Int J Impot Res. 1998; 10:247-250.

17. Zhang HF, Zhang CY, Li XH, et al. Dorsal penile nerves and primary premature ejaculation. Chin Med J (Engl). 2009; 122:3017-3019.

18. Vignoli GC. Premature ejaculation: new electrophysiologic approach. Urology. 1978; 11:81-82.

19. Xin ZC, Choi YD, Rha KH, Choi HK. Somatosensory evoked potentials in patients with primary premature ejaculation. J Urol. 1997; 158:451-455

20. Perelman MA. A new combination treatment for premature ejaculation: a sex therapist's perspective. J Sex Med. 2006; 3:10041012.

21. Dinsmore WW, Hackett G, Goldmeier D, et al. Topical eutectic mixture for premature ejaculation (TEMPE): a novel aerosol-delivery form of lidocaine-prilocaine for treating premature ejaculation. BJU Int. 2007; 99:369-375.

22. Kockott G. Human sexual inadequacy--behavior therapy and the Masters and Johnson technique. Adv Biosci. 1973; 10:219-224.

23. Waldinger MD. Premature ejaculation: state of the art. Urol Clin North Am. 2007; 34:591-599

24. Cooper K, Martyn-St James M, Kaltenthaler E, et al. Interventions to treat premature ejaculation: a systematic review short report. Health Technol Assess. 2015; 19:1-180.

25. Premature ejaculation: Dapoxetine; Nice advice, May 2014.

26. Martyn-St James M, Cooper K, Ren K, et al. Topical anaesthetics for premature ejaculation: a systematic review and meta-analysis. Sex Health. 2015 Nov 25.

27. Chen J, Keren-Paz G, Bar-Yosef Y, Matzkin H. The role of phosphodiesterase type 5 inhibitors in the management of premature ejaculation: a critical analysis of basic science and clinical data. Eur Urol. 2007; 52:1331-9

28. Sansalone S, Russo GI, Mondaini N, et al. A combination of tryptophan, Satureja montana, Tribulus terrestris, Phyllanthus emblica extracts is able to improve sexual quality of life in patient with premature ejaculation Arch Ital Urol Androl. 2016; 88:171176.

29. Huang YP, Chen B, Ping P, et al. The premature ejaculation diagnostic tool (PEDT): linguistic validity of the Chinese version. $J$ Sex Med. 2014; 11:2232-8.

30. Rosen RC, Catania JA, Althof SE, et al. Development and vali- 
dation of four-item version of Male Sexual Health Questionnaire to assess ejaculatory dysfunction. Urology. 2007; 69:805-9.

31. Waldinger MD, Quinn P, Dilleen M, et al. A multinational population survey of intravaginal ejaculation latency time. J Sex Med. $2005 ; 2: 492-7$.

32. U S. Food and Drug Administration. Guidance for Industry Patient Reported Outcome Measures: Use in Medical Product Development to Support Labeling Claims. Federal Register. 2009; 74:65132-3.

33. Gu X, Zhu YZ. Therapeutic applications of organosulfur compounds as novel hydrogen sulfide donors and/or mediators. Expert Rev Clin Pharmacol. 2011; 4:123-33.
34. Das I, Khan NS, Sooranna SR. Potent activation of nitric oxide synthase by garlic: a basis for its therapeutic applications. Curr Med Res Opin. 1995; 13:257-63.

35. Liu K, Zhao W, Gao X, , et al. Diosgenin ameliorates palmitateinduced endothelial dysfunction and insulin resistance via blocking IKKß and IRS-1 pathways. Atherosclerosis. 2012; 223:350-8.

36. Farrell MS, McCorvy JD, Huang XP, et al. In vitro and in vivo characterization of the alkaloid nuciferine. PLoS One. 2016; 11:e0150602.

37. Choi HK, Jung GW, Moon KH, et al. Clinical study of SS-cream in patients with lifelong premature ejaculation. Urology. 2000; $55: 257-261$

\section{Correspondence}

Tommaso Cai, MD (Corresponding Author)

ktommy@libero.it

Department of Urology, Santa Chiara Hospital

Largo Medaglie d'Oro 9, Trento, Italy

Andrea Cocci, MD

Gianmartin Cito, MD

Department of Urology, University of Florence, Florence, Italy

Bruno Giammusso, MD

Department of Andrology, Policlinico Morgagni, Catania, Italy

Alessandro Zucchi, MD

Department of Urology, University of Perugia, Perugia, Italy

Francesco Chiancone, MD

Maurizio Carrino, MD

Department of Urology, Cardarelli Hospital, Naples, Italy

Francesco Mastroeni, MD

Department of Urology, Azienda Ospedaliera Papardo, Messina, Italy

Francesco Comerci, MD

Urologist, Bologna, Italy

Giorgio Franco, MD

Department of Urology, Sapienza University of Rome, Rome, Italy

Alessandro Palmieri, MD

Department of Urology, University of Naples, Federico II, Naples, Italy 